\title{
UNIVERSIDAD Y LUCRO, O EL DILEMA DE LA COBERTURA*
}

\author{
Ricardo D. Paredes \\ Universidad Católica de Chile
}

\begin{abstract}
Mi interpretación de lo que sucedió en Chile desde 1981 (cuando se promulgó la ley que permitió que se crearan universidades privadas sin fines de lucro) hasta hace unos pocos años, fue que hubo, con el objeto de impulsar el desarrollo de la infraestructura y ampliar la cobertura universitaria, un acuerdo tácito de que los distintos factores, en particular el capital necesario para desarrollar la infraestructura, fuesen remunerados.
\end{abstract}

Ahora, ¿cuál es el origen del debate actual sobre el incumplimiento de la norma que prohíbe el lucro? En el trasfondo de este álgido debate está la evidencia palmaria, precisamente, de que desde 1981 ha habido un aumento explosivo de la matrícula universitaria, del que se infiere la siguiente cadena: universidades que remuneran el desarrollo de su infraestructura tienen mayor capacidad de crecer en matrícula; el aumento en la oferta hace disminuir los estándares de aceptación de alumnos; estándares de admisión más bajos hacen aumentar las tasas de deserción y disminuir los estándares académicos, lo que finalmente se traduce en un deterioro de las competencias profesionales de los graduados y titulados. Conforme a esta cadena, el origen de la "masificación" de la educación universitaria está por consiguiente en la remuneración de la infraestructura. Previsiblemente, entonces, la discusión seguirá centrada en cómo evitar que las universidades usen herramientas legales para transferir rentas y en cómo

Ricardo D. Paredes. Profesor Escuela de Ingeniería, Pontificia Universidad Católica de Chile y CEPPE. Dirección electrónica: rparedes@ing.puc.cl.

Agradezco las conversaciones con Rómulo Chumacero, Rodolfo Lauterbach, Valentina Paredes, Sergio Urzúa y Jaime Vatter, y al proyecto CIE01Conicyt, que financia esta línea de investigación. Evidentemente, solo el autor es responsable de las opiniones aquí expresadas.

* Exposición en el Seminario "Lucro y Universidad", realizado el 28 de agosto de 2012 en el CEP. Véanse en esta misma edición las ponencias de Carlos Peña, Carlos Williamson y Arturo Fontaine. (N. del E.) 
obligarlas a reinvertir. Por cierto, es bueno que haya concordancia entre la letra y el espíritu de la ley.

A mi entender, sin embargo, el problema de fondo que afecta al actual sistema universitario en Chile estriba en la precaria formación escolar de los estudiantes que ingresan a la universidad, en la disminución de los requerimientos de admisión y en falta de fiscalización adecuada de la calidad. Estos problemas no derivan necesariamente del hecho de que existan o no universidades con fines de lucro.

\section{Introducción}

Q surgimiento del debate sobre el incumplimiento respecto de la norma que prohíbe el lucro en el sistema universitario chileno el año 2011 tiene distintas explicaciones. Por cierto que no fue la investigación periodística la que descubrió la posibilidad de lucro solo cuando el ministro de Educación de la época reconoció ante las cámaras que vendió su participación en una universidad y que ello le implicó cierta ganancia.

La idea que sostengo en este ensayo es que el legislador, al momento de promulgar la ley, y luego distintas autoridades, legisladores y fiscalizadores, por más de 20 años no consideraron que debían establecerse resguardos especiales en la relación entre la propiedad de las universidades y sus gobiernos corporativos precisamente porque el objetivo de la ley en su forma original fue aceptar el lucro, y concretamente remunerar las inversiones en infraestructura que razonablemente debían estar integradas en la propiedad de las universidades. Ello, con el objeto de impulsar el desarrollo de la infraestructura y la cobertura educacional. Por lo demás, qué diferencia fundamental haría lucrar en una universidad, en un instituto profesional o en un centro de formación técnica, todos entes de la educación superior chilena, de los cuales solo a las universidades les está vedado. Posiblemente, la explicación que permita entender dicha diferencia entre instituciones obedece al pudor de las elites intelectuales que influyeron en la decisión de la ley y cuyas "almas mater" no concebían un sistema universitario de excelencia fuera del esquema tradicional.

Es que de otra forma no existiría explicación para la desidia (intelectual) de académicos y de fiscalizadores, quienes por más de 20 años lo dejaron pasar. Debió existir conciencia de que, al menos como 
posibilidad, detrás del hecho que universidades no fueran dueñas de su infraestructura, sino que se la arrendaran a sociedades poseídas por los mismos controladores, y de las transacciones de propiedad de universidades podría haber intereses económicos y también lucro ${ }^{1}$.

Así, mi interpretación sobre lo álgido y la oportunidad del debate es que, gatillado por una declaración de un ministro de Educación, hay un trasfondo que responde a una coyuntura en la que el aumento explosivo de la cobertura de la educación superior está fuertemente cuestionado, particularmente desde las universidades tradicionales (lo que también ha sido el caso en los EE.UU.), por la existencia de malas universidades, las que explicarían en parte los altos niveles de deserción universitaria. Desde esa evidencia, la crítica y eventualmente la posibilidad de revertir o frenar la expansión ha sido focalizarse en la letra de la ley respecto de la prohibición de lucro. La vinculación es así casi directa y un buen analista pudo preverla: universidades que remuneran el desarrollo de su infraestructura tienen mayor capacidad de crecer en matrícula; ellas dan cuenta de la masificación, que dado el sistema escolar vigente requiere ir reduciendo los estándares de aceptación de alumnos; ello deriva en mayores dificultades de retención y en el deterioro del status profesional, lo que sumado a la falta de vigilancia y control sobre la calidad permite finalmente cuestionar la masificación y el instrumento más directo que la permitió.

En este ensayo analizo los elementos que, a mi juicio, son fundamentales en la discusión sobre el lucro en las universidades en Chile, centrándome en las consecuencias prácticas de la discusión y de la prohibición.

\section{Lucro desde una perspectiva económica}

El lucro en economía es sinónimo de ganancia, detrás de la cual no hay acepción negativa. Está también en la acepción legal; lucro cesante, por ejemplo, puede decir relación con lo que demanda un taxista cuando su automóvil es chocado por un conductor ebrio y ha debido dejar de trabajar. En este caso, se trata de la ganancia que el taxista legítimamente pudo obtener por su trabajo. Podemos asociar así a lucro,

1 Cada uno de estos elementos no demuestra lucro. Así, por ejemplo, algunas transacciones se han hecho sobre sociedades que incluyen, además de universidades, otras sociedades que sí pueden lucrar; la integración vertical inmobiliarias-universidades tiene también sentido desde la perspectiva de la eficiencia. 
la ganancia, el salario, o el pago por el otorgamiento de un servicio, sin que necesitemos asociarlo con una ganancia ilegítima o excesiva.

La aproximación económica a diversos temas se centra en las consecuencias de las acciones (economía positiva) y no en lo que "deber ser". Así, respecto del hecho de que muchas personas persigan lucrar la economía no tiene instrumental para responder. Por ejemplo, la economía no responde cuánto más justa sería la sociedad si es que las personas fueran más generosas o quisieran donar su tiempo y trabajo, lo que por cierto hace diferente una sociedad. Sí, desde las primeras lecciones de economía, los estudiantes aprenden sobre las consecuencias de que las personas busquen su propio interés, lo que puede resumirse en la siguiente afirmación de Adam Smith, en su libro La Riqueza de las Naciones (1776):

No es de la benevolencia del carnicero, del cervecero o del panadero en la que debiéramos confiar para tener nuestra cena, sino que de la búsqueda de su propio interés.

Desde la perspectiva de política pública, esta frase se traduce en que cuando la caridad no es suficiente para alimentar o educar, entonces el mismo egoísmo, el propio interés, puede ser un motor para hacerlo. Ello, claramente, también es objeto de controversia no solo desde la práctica y de los efectos, sino que está en el centro de la discusión sobre las libertades.

Visiones diferenciadas respecto del rol del lucro en torno a la educación se pueden extrapolar del diálogo sostenido por el general William Westmoreland y Milton Friedman ante la Comisión Presidencial Nixon, cuyo objetivo era analizar el fin del reclutamiento obligatorio en los EE. UU. y sustituirlo por una fuerza militar completamente pagada. El diagnóstico de Friedman era que como no bastaba con el número de voluntarios dispuestos a hacer el servicio militar sin que mediaran pagos, era necesario pagar un salario de modo de generar un contingente suficiente (voluntario), sin requerir el reclutamiento forzoso.

General: No me gustaría comandar un ejército de mercenarios...

Friedman: ¿Es que acaso preferiría comandar una fuerza de esclavos? 
General: No me gusta oír que alguien se refiere a nuestros patriotas reclutas como esclavos.

Friedman: No me gusta oír que alguien se refiera a nuestros patriotas voluntarios como mercenarios. Si ellos son mercenarios, entonces yo, señor, soy un profesor mercenario y Ud., señor, un general mercenario. Somos servidos por médicos mercenarios, usamos abogados mercenarios y obtenemos nuestra comida de carniceros mercenarios ${ }^{2}$.

Así, desde Smith a nuestros tiempos, la idea que la búsqueda de ganancias o lucro hace actuar a las personas y que dichas ganancias están alineadas o pueden alinearse con los objetivos de la sociedad, constituye una piedra angular de la política pública, pues en este sentido las acciones seguidas por el egoísmo compiten, al menos conceptualmente, con la acción directa del Estado. La ganancia, el beneficio, o el lucro, deben entenderse como el pago que induce, para que quienes no son generosos, aporten recursos y esfuerzo.

\section{Lucro normal y la oferta de generosos}

Ciertos niveles de ganancia o lucro podrán superar lo necesario para inducir al aporte de los insumos que poseen las personas y sobre eso, sobre el lucro excesivo, la teoría económica también tiene algo que decir. Las rentas ricardianas, vinculadas literalmente en David Ricardo a la tierra, pero perfectamente extrapolables a cualquier recurso naturalmente escaso, como pudiera ser la capacidad de innovar, de gestionar, o de asumir nuevos proyectos, constituyen exceso de lucro, en el sentido que pueden ser eliminadas sin inducir a los dueños de los factores a entregar menor esfuerzo o aportar menos insumos productivos. Mientras las rentas ricardianas no reducen el aporte de insumos y esfuerzo, las rentas monopólicas sí lo hacen. Sin embargo, la discusión económica sobre el lucro o la ganancia no es sobre excesos, porque sobre estos últimos la teoría económica tiene consensos y las leyes dan o pueden dar cuenta directamente a través de sistemas de impuestos y de la legislación antimonopolios.

2 http://delong.typepad.com/sdj/2012/01/econ-1-uc-berkeley-spring2012-why-we-are-reading-milton-friedman-and-rose-director-friedman-free-tochoose.html. 
La discusión pertinente al lucro en educación es sobre la ganancia normal, aquella de la que la evidencia sugiere que mayoritariamente las personas exigen como retribución por el aporte que entregan. Y si lucro es sinónimo de remuneración necesaria para inducir en algunos el aporte de insumos para generar educación, incluyendo el pago a los sostenedores educacionales, entonces la controversia está erróneamente centrada en el lucro excesivo, el innecesario para que los dueños de los insumos aporten al proceso educacional.

A partir de esta ley, los recursos públicos entregados a las entidades educativas deberán ser destinados precisamente al cumplimiento de la función educativa, porque para eso son los recursos públicos, para ser invertidos en educación y nada más que educación (...). Y vamos a fiscalizar que ello suceda. (Extracto del discurso de la Presidenta Bachelet con ocasión del envío del proyecto de ley que ponía fin a las escuelas con fines de lucro, abril 2007).

Compartiendo el propósito de que los recursos entregados por el Estado deben estar estrictamente orientados a mejorar la educación, dado un esfuerzo estatal, lo que por cierto es objeto de debate, la afirmación previa sugiere ya sea que: i) las instituciones con fines de lucro obtienen ganancias que exceden las necesarias para inducir voluntariamente los aportes de recursos que hacen para entregar una educación de calidad; ii) que es posible cubrir las necesidades de cobertura educativa solo con instituciones sin fines de lucro, o que iii) intrínsecamente, la naturaleza jurídica hace una diferencia en la calidad de la educación que cada una provea o en los costos que la educación le significan al Estado.

Sobre el primer aspecto, exceso de lucro, ya señalamos que hay instituciones que están derechamente orientadas a ese efecto y, más aún, el tema específico del exceso no es parte de la controversia. Sobre los otros dos puntos nos referimos en lo que sigue.

\subsection{Oferta de instituciones sin fines de lucro}

Un error lógico recurrente surge de comparar instituciones con y sin fines de lucro. Como existen algunas personas e instituciones que, con intereses diversos, no requieren remuneración por el aporte que hacen, por ejemplo donando su trabajo o los edificios universitarios, 
entonces pudiéramos deducir que prohibiendo las instituciones con fines de lucro (las que requieren remuneración) obtendremos educación más barata y de mejor calidad. Pero ello será cierto solo si es que hay suficiente oferta de instituciones educativas sin fines de lucro, de modo que ellas satisfagan la demanda por educación. Si la oferta de personas o instituciones generosas no es suficiente para las necesidades de cobertura educativa que definimos como sociedad, necesitaremos de otras formas de proveer educación, y lo que desde la política pública de mediano plazo es evidente, es que la cobertura la hagan las instituciones del Estado, o que éste induzca, mediante subsidios, el incremento de la cobertura por parte de instituciones sin fines de lucro (cambiar las actitudes y generosidad de las personas pudiera ser posible, pero debe tomar más tiempo).

Pero ¿por qué necesitaría una institución sin fines de lucro un subsidio estatal para aumentar su cobertura? El motivo es evidente, si la oferta de generosos no es suficiente, necesitará financiar la nueva infraestructura, los equipos de gestión que el contingente adicional de estudiantes demande, etc. ¿Y si es el Estado el que directamente, en sus propias universidades, provee dichos cupos? Soslayando la discusión sobre la selectividad que las universidades más tradicionales quisieran mantener, tampoco se puede eludir el hecho de que también el Estado requerirá del aporte de insumos, de factores productivos, que no están a libre disposición. Esto es, si es el Estado el que educa directamente, el Estado debe contratar profesores, infraestructura, gestión, y esos recursos tampoco están disponibles para él sin la compensación adecuada, o al menos no en la cantidad suficiente. En este caso, formalmente habrá menos instituciones con fines de lucro, pero no se ahorrarán recursos para la educación.

\section{2 - ¿Son las instituciones sin fines de lucro lo que la sociedad desea?}

El planteamiento anti lucro sugiere desvío de recursos de los propósitos de la educación, lo que descartando el lucro excesivo implicaría una orientación anti educación. Es decir, los recursos obtenidos servirían para el pago justo de los insumos educativos, pero además existirían pagos (o retiros) que no remuneran lo que es necesario para contribuir exactamente a la educación de calidad que la sociedad demanda. 
Es cierto que algunas personas o grupos están dispuestos a entregar sus servicios, como la gestión, e incluso a donar infraestructura sin remuneración. Algunos lo hacen por el amor a la educación, otros porque creen que a través de la educación pueden transmitir valores que les son propios, sin perjuicio de que dichos valores no tienen por qué ser concordantes con los deseados por el Estado. Consecuentemente, es posible que no haya diferencias cuando hablamos de lucro y no lucro. Cuando un grupo quiere transmitir sus valores religiosos o políticopartidistas, cuando quienes toman las decisiones de matrícula y aranceles obtienen de sus propios salarios remuneraciones mayores que las del mercado a través de una institución que formalmente no lucra, o cuando lo que serían excedentes se destinan a inmuebles o construcciones localizadas en lugares de alto valor de la tierra, no hay en principio diferencias desde la perspectiva educacional de si el destino de los excedentes fuera retirado por los gestores.

La diferencia entre instituciones sobre lo que hacen con los excedentes, es decir, la diferencia entre ingresos y gastos después de haber pagado todos los insumos, y que parece haber focalizado la discusión, es fundamentalmente contable, no de fondo. El sentido económico del excedente se refiere a lo que debiera quedar después de haber incurrido en el costo necesario (sentido de costo económico) para proveer educación de calidad. Por ello, desde el punto de vista económico y educativo, no hace diferencia entre una universidad en la que los dueños retiran los excedentes para financiar vacaciones en el Caribe, de otra que paga por factores que no son necesarios para el desarrollo educacional.

Como debe quedar meridianamente claro, ni siquiera la diferencia se puede establecer respecto de instituciones estatales. Tampoco el argumento debiera centrarse en las declaraciones de principio, que poca relación pueden tener con lo que se hace. Si el argumento es que las instituciones con fines de lucro (o las privadas) no tienen valores republicanos o no tienen los valores que el Estado considera que debieran tener, entonces quedamos atrapados en una discusión en la que difícilmente se puede aportar desde la perspectiva económica, sin perjuicio de que tal diferencia de valores sea de alguna manera medible. Y de hecho, también sobre ello hay controversia. Al respecto, y solo como contraste con la idealización de ciertos tipos de escuela o educación, un debate antiguo en el mundo no sugiere una tipología necesariamente más concordante con esos principios y hay claridad en el riesgo de captura de los principios 
idealizados no solo en dictaduras, sino también en contextos de gobiernos democráticos, en camino o en riesgo de transición al totalitarismo ${ }^{3}$.

En esta línea, Chumacero y Paredes (2009) describen la interpretación de Lott (2007) respecto de la génesis de la educación pública en los EE. UU., la que trasluce una visión muy lejos del ideal:

Hacia los años 1820, los legisladores en Nueva York estaban preocupados por el tipo de educación que estaban recibiendo los niños, porque la mayoría de las escuelas (privadas) eran dirigidas por la Iglesia Católica. Con ello, decidieron subsidiar las escuelas protestantes a través de sistemas similares a vouchers. Sin embargo, para atraer más estudiantes a las nuevas escuelas protestantes, ellas comenzaron a enseñar lo que la mayoría de los padres aparentemente deseaban se les enseñara a sus hijos (a leer, escribir y operaciones matemáticas) y a enseñar menos de lo que no querían (entrenamiento protestante). En la medida que los legisladores vieron que su objetivo no se cumplía, progresivamente fueron impulsando que fuera el Estado el que implementara y dirigiera escuelas, lo que ha llevado a algunos observadores a concluir que la educación pública tiene más que ver con adoctrinamiento. Por ejemplo, Lott (1999) muestra que bajo regímenes totalitarios (que son los que tienen los mayores retornos al adoctrinamiento), es más probable hallar mayores niveles de gasto en educación ${ }^{4}$.

\section{Conclusiones}

Lo que hubo en Chile no parece haber sido un error legislativo ni de fiscalización posterior, sino un propósito claro de permitir que los distintos factores, particularmente el capital necesario para desarrollar la infraestructura, fuera remunerado. No proveyéndola el Estado mediante su intervención directa, si no se hubiera permitido la remuneración de los

3 Véase, por ejemplo, en el caso de Chile, en 1972: "Un Sistema Nacional para la Educación Permanente en una Sociedad de Transición al Socialismo" 1973. Argentina: "Cristina Defiende Militancia Política en las Escuelas (La Cámpora)", agosto, 2012, y para Venezuela: http://otogran.com.over-blog. es/article-educacion-venezolana-en-el-contexto-de-la-revolucion-bolivariana-yla-democracia-del-siglo-xxi-66826819.html.

${ }^{4}$ R. Chumacero y R. Paredes: "Should for Profit Schools Be Banned?", 2009; Lott, J., Freedomnomics: Why the Free Market Works and Other Half Baked Theories Don't, 2007. 
distintos factores, habría sido inviable el crecimiento de infraestructura y el aumento de oferta universitaria. Si ello fue o no una buena idea es otra cosa, y posiblemente, a juzgar por el aumento de oferta de cupos, la reducción en los requerimientos de calificación previa de los estudiantes para llenar esos cupos y la falta de fiscalización adecuada respecto de la calidad, no lo fue.

Sin embargo, el origen de ese problema no es la existencia o no de instituciones con fines de lucro, sino fundamentalmente el hecho de que la mayor cobertura se ha inducido sin restricciones a la formación previa, lo que hace que difícilmente, aunque los alumnos logren superar algunas de sus carencias, puedan llegarse a satisfacer las expectativas de quienes ingresan a la educación superior.

Lo que viene es, previsiblemente, que seguirá centrada la discusión en cómo controlar el cumplimiento de la letra de la ley, en cómo evitar que las universidades usen herramientas legales para transferir rentas y en obligarlas a una reinversión. Ello tiene ventajas si se piensa que es bueno disponer de leyes que mantengan consistencia entre espíritu y letra, pero mi interpretación de lo que ha habido en Chile desde 1980, e ininterrumpidamente hasta hace menos de tres años, es un acuerdo tácito en el sentido de que el pago por la inversión, por los aportes de capital, debían ser remunerados, esto es, que en la acepción económica, no jurídica ni contable, el lucro es funcional al aumento de oferta educativa.

Las consecuencias de "cambiar" las reglas del juego, moviéndonos al nuevo consenso sobre aplicar fielmente las reglas que permitan terminar con el lucro en las universidades serán presumiblemente menores en Chile. Es previsible que a las universidades se les exija vender sus inmobiliarias, y en ausencia de usos alternativos reales para ellas se abra un debate enorme sobre los precios de venta, debates sobre lo expropiatorio que pueden llegar a ser, sobre la constitucionalidad, etc. Es menos previsible que en este caso, por la magnitud de los proyectos y por su especificidad, la cobertura se revierta o cambie el destino que hoy tienen - el universitario-, lo que no ocurriría en el caso de una reforma en el mismo sentido de la educación escolar. Sin embargo, aun así, y con propósitos ya no de predecir lo que viene sino muy especialmente de extraer lecciones del caso chileno, cabe hacer el ejercicio conceptual sobre qué educación tendría Chile si es que efectivamente, sin mayor generosidad ni mayor intervención estatal, hubiéramos impedido que existieran las instituciones que hoy están bajo sospecha de lucrar. 
FIGURA $N^{\circ} 1$

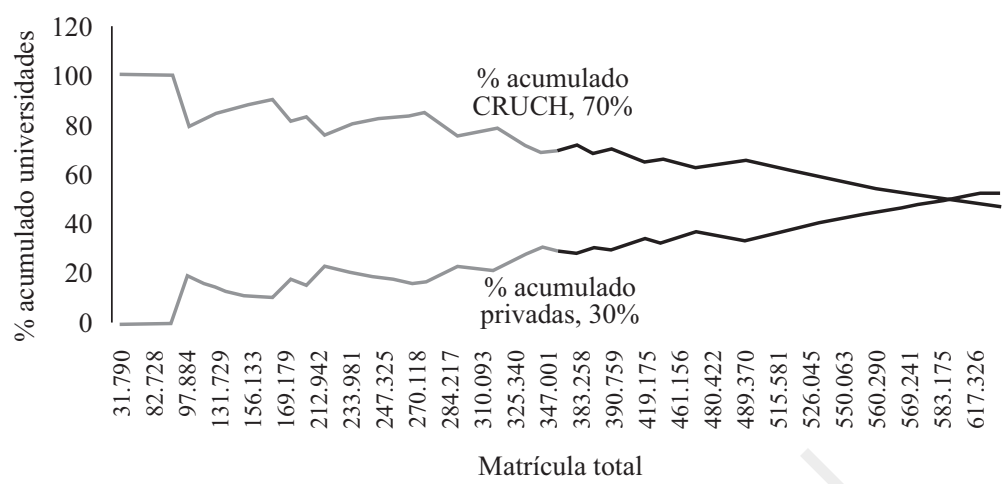

Fuente: R. Paredes, C. Valdivia y A. Barrios (2012): "Calidad de las Universidades en Chile: Rankings, Categorías y Política Pública”, Documento de Trabajo, Departamento de Ingeniería Industrial, PUC.

La Figura $\mathrm{N}^{\circ} 1$, tomada del trabajo de Paredes, Valdivia y Barrios (2012), ilustra el tipo de consecuencias. En el trabajo aludido, y a partir de todas las variables asociadas a calidad universitaria disponibles en Chile y que se usan en los principales rankings de calidad en el mundo, construyen un ranking único y ordenan, de "mejor a peor", las universidades según la matrícula que cubren. Luego, arbitrariamente definimos un grado de selectividad mayor que el que hoy existe, por ejemplo $50 \%$. La figura muestra que si termináramos con las "peores universidades", calificadas según el ranking señalado que sirven el 50\% de los alumnos, en ese caso, el $70 \%$ de los alumnos iría a una universidad del CRUCH y el 30\% iría a universidades privadas creadas con posterioridad a 1980 . Esto es, un sistema más selectivo en calidad estaría más representado por universidades tradicionales que lo que está hoy, pero que si de calidad se trata, no convendría hacer un corte del tipo CRUCH-no CRUCH. Adicionalmente, sabemos que de entre las universidades privadas que prevalecerían por el corte de criterio de calidad, algunas de ellas han sido cuestionadas respecto de su efectiva orientación al lucro.

En síntesis, la calidad del sistema universitario en Chile hoy se basa en universidades tradicionales sin fines de lucro y, también, en otras universidades privadas creadas mayoritariamente después de 1980, de las que no se ha cuestionado su vocación de "no lucro". Hay, sin embargo, algunas que han sido cuestionadas como elusoras de la legislación que prohíbe el lucro, pero que de haberse prescindido de ellas habría habido consecuencias adversas en la calidad del sistema chileno. 


\section{REFERENCIAS BIBLIOGRÁFICAS}

Bachelet, Michelle. Discurso con ocasión del envío del proyecto de ley que ponía fin a las escuelas con fines de lucro, abril 2007.

Comisión Presidencial Nixon. Diálogo entre el general William Westmoreland y Milton Friedman. En http://delong.typepad.com/sdj/2012/01/econ-1-ucberkeley-spring-2012-why-we-are-reading-milton-friedman-and-rosedirector-friedman-free-to-choose.html.

Chumacero, R. y R. Paredes. "Should for Profit Schools Be Banned?". MPRA Paper 15599 (2009). En http://mpra.ub.uni-muenchen.de/15099/.

Lott, John. R. Freedomnomics: Why the Free Market Works and Other HalfBaked Theories Don't. Washington, D.C.: Regnery, 2007.

Paredes, R., C. Valdivia y A. Barrios. "Calidad de las Universidades en Chile: Rankings, Categorías y Política Pública”. Documento de Trabajo, Departamento de Ingeniería Industrial, Pontificia Universidad Católica de Chile, 2012.

Smith, Adam. La Riqueza de las Naciones [1776]. Longseller S.A., 2001.

\section{Documentos:}

Argentina: "Cristina Defiende Militancia Política en las Escuelas (La Cámpora)", agosto, 2012. En http://www.mer.cl/Pages/NewsDetail.aspx?dt=2012-0826\&PaginaId=4\&bodyid $=1$.

Chile: "Un Sistema Nacional para la Educación Permanente en una Sociedad de Transición al Socialismo" [1972]. En La Crisis Educacional. Santiago: Editorial Quimantú, 1973. [http://es.scribd.com/doc/61761598/UnidadPopular-La-crisis-educacional-y-la-Escuela-Nacional-Unificada-1973.]

Venezuela: http://otogran.com.over-blog.es/article-educacion-venezolana-enel-contexto-de-la-revolucion-bolivariana-y-la-democracia-del-sigloxxi-66826819.html. 